\title{
Synthesis of porous poly(acrylamide) hydrogels using calcium carbonate and its application for slow release of potassium nitrate
}

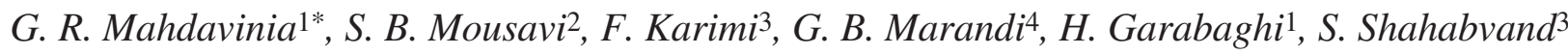 \\ ${ }^{1}$ Department of Chemistry, Faculty of Science, University of Maragheh, P.O. Box 55181-83111, Maragheh, Iran \\ ${ }^{2}$ Department of Soil Science, Faculty of Agriculture, University of Maragheh, P.O. Box 55181-83111, Maragheh eh, Iran \\ ${ }^{3}$ Department of Biotechnology, Faculty of Science, University of Maragheh, P.O. Box 55181-83111, Maragheh eh, Iran \\ ${ }^{4}$ Department of Chemistry, Islamic Azad University, Karaj Branch, P. O. Box 31485-313, Karaj, Iran
}

Received 3 January 2009; accepted in revised form 3 March 2009

\begin{abstract}
Porous poly(acrylamide) was synthesized using calcium carbonate microparticles and subsequent acid treatment to remove the calcium carbonate. Methylenebisacrylamide and ammonium persulfate/sodium metabisulfite were used as crosslinking agent and redox initiator, respectively. The porous structure of resulted hydrogels was confirmed using SEM micrographs. The effect of methylenebisacrylamide concentration and calcium carbonate amount on the swelling of the hydrogels was investigated. The results showed that the effect of methylenebisacrylamide and calcium carbonate variables on the swelling is reverse. The hydrogels were subsequently utilized for the loading of potassium nitrate. Potassium nitrate as active agent was loaded into hydrogels and subsequently the release of this active agent was investigated. In these series of investigation, the effect of content of loading, methylenebisacrylamide and calcium carbonate amount on the release of potassium nitrate from hydrogels was investigated.
\end{abstract}

Keywords: polymer gels, porous polyacrylamide, swelling, slow release, potassium nitrate

\section{Introduction}

Hydrogels are three-dimensional hydrophilic polymer networks capable of imbibing large amount of water [1]. For this reason they have been used widely in the field of drug delivery, immobilization of enzymes, dewatering of protein solutions, solute separation, baby diapers, soil for agriculture and horticulture, water-blocking tapes, absorbent pads, and numerous other applications [2-8]. High swelling rate is an important property of hydrogels. They mainly need several hours to reach maximum absorption capacity. The slow swelling of dried hydrogels is due to the slow diffusion of water into the glassy matrix of the dried hydrogels [9]. Chen and Park developed a new kind of superabsorbent polymers, so called superporous hydrogels [10]. There are several well known methods to prepare porous hydrogels, including phase separation, foaming technique, emulsion-template synthesis, and particulate leaching and freeze-drying [11, 12]. Poly(acrylamide) (PAAm) is an important and hydrophilic polymer for preparation of hydrogels [13]. The common method to synthesis of PAAm hydrogels is the free radical crosslinking copolymerization of AAm monomer with multifunctional vinyl monomers [13]. PAAm hydrogels and their derivatives are the subject of many studies $[14,15]$. PAAm hydrogels have proven capability of water absorption and biocompatibility with physiologic body fluids. The application of PAAm hydrogels in 
controlled release of agrochemicals and bioactive have been investigated [16, 17]. Researchers have been reported porous PAAm hydrogels [18-20].

Synthesizing porous hydrogels using solution polymerization method in the presence of porogen (that produce gas bubbles from reaction of porogen with chemicals) is a difficult task, because produced gas bubbles from porogen leave the solution easily. The main aim of this work was to synthesize porous PAAm hydrogels through solution polymerization and its application to slow release of agrochemicals. To the best of our knowledge, there have been no previous reports on using the calcium carbonate for synthesis of porous PAAm hydrogel. The leaching of calcium carbonate and subsequently evolving of $\mathrm{CO}_{2}$ gas from hydrogel matrix results in porous structure. Potassium nitrate, an agrochemical agent was loaded in the hydrogels and the release of this active agent in deionized water was studied.

\section{Experimental}

\subsection{Materials}

Acrylamide (AAm, Merck Co.) was used after crystallization from acetone. Methylenebisacrylamide (MBA, Fluka Co.), ammonium persulfate (APS, Fluka Co.), and sodium metabisulfite (SMBS, Fluka Co.) were used as received. Calcium carbonate $(\mathrm{CCb} \sim 3 \mu \mathrm{m}$, Fluka Co.), and other chemicals were used as received.

\subsection{Synthesis of PAAm hydrogel}

AAm solution was prepared in a one-liter reactor equipped with mechanical stirrer and gas inlet. AAm was dissolved in degassed distilled water. Reaction variables are given in Table 1. In general, AAm ( $3.0 \mathrm{~g}, 42 \mathrm{mmol})$ was dissolved in $30.0 \mathrm{ml}$ of distilled degassed water. MBA as a crosslinker $(0.050 \mathrm{~g}$ in $2 \mathrm{ml}$ water, $0.32 \mathrm{mmol})$ was added to the AAm solution and the mixture was continuously stirred under nitrogen gas. Various amounts of calcium carbonate powder (1.5, 3 and $6 \mathrm{~g}$ ) were added to the reaction mixture and allowed to stir for $10 \mathrm{~min}$. Then APS $(0.05 \mathrm{~g}$ dissolved in $2 \mathrm{ml}$ water, $0.22 \mathrm{mmol})$ and SMBS (0.05 $\mathrm{g}$ dissolved in $2 \mathrm{ml}$ water, $0.26 \mathrm{mmol}$ ) as a redox initiator were added to the solution and stirred for $30 \mathrm{~min}$. Obtained hydrogels in bulk state were cut in similar pieces $(\sim 2 \times 2 \times 2 \mathrm{~mm})$. Subsequently, the calcium carbonate particles were dissolved in the aqueous $10 \% \mathrm{wt}$ $\mathrm{HCl}$ solution by immersion of hydrogels for $24 \mathrm{~h}$ at ambient temperature. For removing the calcium chloride from dissolution of calcium carbonate and other remained substrates, the hydrogels were immersed in excess amount of deionized water for 6 days with daily refreshment of the water. In order to assure the complete removal of $\mathrm{Ca}^{2+}$ and $\mathrm{Cl}^{-}$ ions, $\mathrm{CO}_{3}^{2-}$ and $\mathrm{Ag}^{+}$ions were used, respectively; in which lack of any precipitation was attributed to a complete removal. After this time, the hydrogels were poured to $300 \mathrm{ml}$ ethanol. After dewatering for $24 \mathrm{~h}$, the hardened hydrogel products were filtered, washed with fresh ethanol $(2 \times 50 \mathrm{ml})$ and dried at $50^{\circ} \mathrm{C}$ until obtaining a constant weight.

\subsection{Bulk density measurement}

Bulk density of dried hydrogels was determined by using picnometric method outlined in ASTM D792. In general, $1 \mathrm{~g}$ of dried hydrogels was placed in a $10 \mathrm{ml}$ picnometer with known weight. Then the picnometer containing sample was filled with acetone as non-solvent and picnometer containing sample and acetone weighed to determine the density of sample according to standard method.

\subsection{Swelling studies}

Dried hydrogel pieces were used to determine the degree of swelling. The degree of swelling $(D S)$

Table 1. Amount of reaction parameters for synthesis of porous hydrogels $\left(\mathrm{H}_{2} \mathrm{O} 40 \mathrm{ml}\right.$, APS/SMBS 0.22/0.26 mmol)

\begin{tabular}{|c|c|c|c|c|c|}
\hline Run & AAm [mmol] & MBA [mmol] & CaCO3 [g] & DS [g/g] & loaded of PN [\%] \\
\hline SP1 & 42 & 0.32 & 0.0 & 24 & 28.0 \\
\hline SP2 & 42 & 0.32 & 1.5 & 34 & - \\
\hline SP3 & 42 & 0.32 & 6.0 & 44 \\
\hline SP4 & 42 & 0.32 & 3.0 & 41 \\
\hline MBA1 & 42 & 0.16 & 3.0 & 34.0 & 51.7 \\
\hline MBA2 & 42 & 0.32 & 3.0 & 56.1 \\
\hline
\end{tabular}


was determined by immersing the hydrogels $(0.1 \mathrm{~g})$ in distilled water $(100 \mathrm{ml})$ and was allowed to soak for $24 \mathrm{~h}$ at room temperature. After this time, they were removed from the water, blotted with filter paper to remove surface water, weighed and the $D S$ was calculated using Equation (1):

$$
D S=\frac{W_{s}-W_{d}}{W_{d}}
$$

where $W_{s}$ and $W_{d}$ are the weights of the samples swollen in water and in dry state, respectively. For studying the swelling kinetics of the hydrogels, a certain amount of samples $(0.20 \mathrm{~g})$ was poured into numbers of weighed tea bags and was immersed in $200 \mathrm{ml}$ distilled water. At consecutive time intervals, the water absorbency of the hydrogels was measured according to the above method.

\subsection{Loading/release of potassium nitrate}

The potassium nitrate (PN) was dissolved in $25 \mathrm{ml}$ deionized water with desired concentration $(1 \% \mathrm{wt}$ of PN for all samples, but in the series of effect of \%loading on the release of PN, 0.5, 1 and $2 \%$ wt of PN solution was used). Pre-weighed dried and purified hydrogel pieces $(0.1 \mathrm{~g})$ were immersed in it for $24 \mathrm{~h}$ at room temperature. After withdrawing the loaded hydrogels, the volume of the remained PN solutions was filled to $25 \mathrm{ml}$ and according to conductivity of these diluted solutions and calibration curve, the amount of loaded PN was calculated. Loaded hydrogels were filtered and dried in an oven at $40^{\circ} \mathrm{C}$ for constant weight. The milligram of loaded PN in hydrogels was calculated using calibration curve. The amounts of loaded PN in hydrogels were given in Table 1. For study the release of $\mathrm{PN}$, deionized water was chosen as a release medium. In general, dried $\mathrm{KNO}_{3}$ loaded hydrogels were immersed in $100 \mathrm{ml}$ deionized water under un-stirred condition. At intervals, the amount of PN released was evaluated using conductometer. The released PN was determined from the calibration curve.

\subsection{Instruments}

The surface morphology of the hydrogels was examined using scanning electron microscopy (SEM). The purified hydrogels were dewatered and dried at $40^{\circ} \mathrm{C}$. The dried hydrogels were grinded and sieved to obtain 40-60 mesh. Dried hydrogel powder with 40-60 mesh size were coated with a thin layer of palladium gold alloy and imaged in a SEM instrument (Leo, 1455 VP). A conductivity meter (HANNAN, HI 8819N) was used to study of release of potassium nitrate.

\section{Results and discussions}

\subsection{Synthesis and characterization}

The aim of this study was to produce a porous crosslinked-PAAm hydrogel under solution polymerization. Porous hydrogel was successfully prepared by the incorporation of $\mathrm{CaCO}_{3}$ microparticles. In the synthesized hydrogels, the porous structure can be formed in two ways: (a) acid treatment to leaching the $\mathrm{CaCO}_{3}$ particles and (b) the hydrogel containing $\mathrm{CaCO}_{3}$ particles has a pasty and jelly state that prevents removing the evolved $\mathrm{CO}_{2}$ gas (from neutralization of $\mathrm{CaCO}_{3}$ in the presence of acid) from the pasty and jelly state. So, the removed gas creates pores in the hydrogel. Figure 1 indicates SEM micrographs of hydrogels. The PAAm as a hydrogel component and $\mathrm{CaCO}_{3}$ particles were observed in the hybrids, as shown in Figure 1b. After $\mathrm{HCl}$ treatment, the $\mathrm{CaCO}_{3}$ particles have disappeared and pores could be clearly observed in the hydrogel, as shown in Figure 1c. On the other hand, pores were not observed in hydrogels prepared without the $\mathrm{CaCO}_{3}$ particles, as shown in Fig-
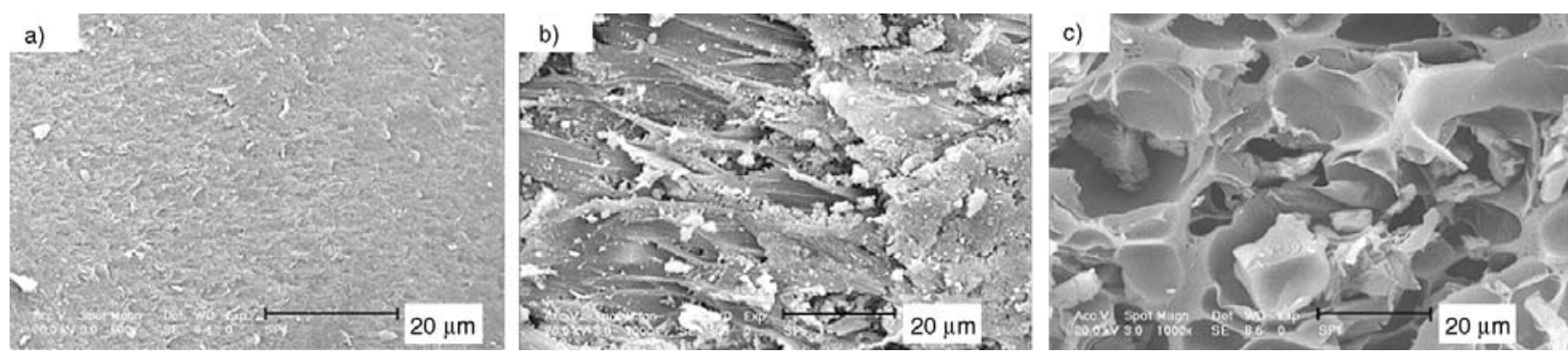

Figure 1. SEM micrographs $(\times 1000$, Scale bar: $20 \mu \mathrm{m})$ of a) non-porous PAAm, b) PAAm hydrogel containing $\mathrm{CaCO}_{3}$ and c) porous hydrogel after removing of $\mathrm{CaCO}_{3}$ 
ure 1a. These observations indicate that porous PAAm hydrogels were successfully prepared by the present technique.

\subsection{Effect of $\mathrm{CCb}$ content on porosity}

For all dried hydrogels, the bulk density of dried hydrogels was determined using picnometer. As shown in Table 2, the increase in $\mathrm{CaCO}_{3}$ content has a big influence on the density of the hydrogels. The results reveal that the increase in $\mathrm{CaCO}_{3}$ causes a decrease in the density of the hydrogels. Decrease in density can be attributed to the pores containing air [21]. Using the high content of $\mathrm{CaCO}_{3}$ to synthesize of hydrogel causes the high number of produced pores, and subsequently the density will be decreased. So, the decrease in density can be attributed to the increase in porosity.

Table 2. Effect of $\mathrm{CaCO}_{3}$ content on the bulk density ( $\rho$ ) of dried hydrogels

\begin{tabular}{|c|c|c|}
\hline Sample & $\mathbf{C a C O}_{\mathbf{3}}[\mathbf{g}]$ & $\boldsymbol{\rho}\left[\mathbf{g} / \mathbf{c m}^{\mathbf{3}}\right]$ \\
\hline SP1 & 0.0 & 1.64 \\
\hline SP2 & 1.5 & 1.23 \\
\hline SP3 & 3.0 & 1.02 \\
\hline SP4 & 6.0 & 0.85 \\
\hline
\end{tabular}

\subsection{Swelling study}

\subsubsection{Effect of $\mathrm{CaCO}_{3}$ content on swelling}

Figure 2 illustrates the equilibrium of water content and dynamic swelling behavior of hydrogels as a function of the $\mathrm{CaCO}_{3}$ amount. As presented in this figure, by using the $\mathrm{CaCO}_{3}$ in the feed mixture, the water absorbency of the hydrogels increases. This may be attributed to the fact that using $\mathrm{CaCO}_{3}$ in

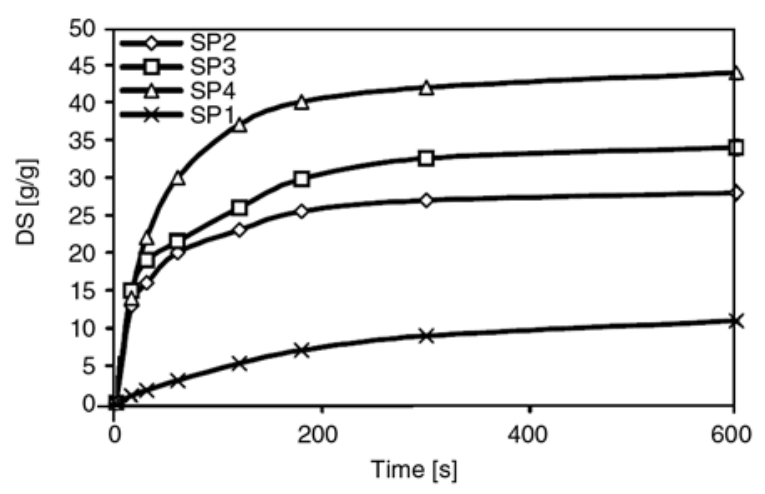

Figure 2. Water absorbing and dynamic swelling dependency of porous PAAm hydrogel as a function of amount of $\mathrm{CaCO}_{3}$ the hydrogel makes pores in the hydrogel structure and results in a high water uptake. Also, hydrogels absorb higher amount of water with increasing $\mathrm{CaCO}_{3}$ amount in reaction feed that may be due to increase in pore number. Also, the effect of $\mathrm{CaCO}_{3}$ content on the swelling kinetic of the hydrogels was investigated. A preliminary study was conducted on the hydrogels swelling kinetics. The rate of water uptake in porous hydrogels is higher than that of non-porous hydrogel. Increase in the rate of absorption would be expected from the increase in surface area with increasing porosity of hydrogels. The swelling-time data can be fitted with the Equation (2) [22] to derive the power and rate parameters:

$S_{t}=S_{e}\left(1-e^{-t / \tau}\right)$

where $S_{t}$ is swelling at time $t[\mathrm{~g} / \mathrm{g}], S_{e}$ is equilibrium swelling ('power parameter', $[\mathrm{g} / \mathrm{g}]$ ), $t$ is time [sec] for swelling $S_{t}$, and $\tau$ stand for 'rate parameter' (time for $S_{\tau}$ ), [s]. The rate parameters for hydrogels prepared with using $0,1.5,3$ and $6 \mathrm{~g}$ of $\mathrm{CaCO}_{3}$ are $500,128,113$, and $100 \mathrm{sec}$, respectively. The $\tau$ value for hydrogel with no- $\mathrm{CaCO}_{3}$ is higher than those in the samples was synthesized using $\mathrm{CaCO}_{3}$. This may simply be attributed to the fact that with using $\mathrm{CaCO}_{3}$ in synthesis of hydrogel the porous structure is formed.

\subsubsection{Effect of MBA concentration}

Crosslinks are necessary to form a hydrogel in order to prevent dissolution of the hydrophilic polymer chains in an aqueous environment. To investigate the effect of MBA concentration on the water absorbency of the porous PAAm, the crosslinker concentration was chosen $0.13,0.32$ and $0.65 \mathrm{mmol}$. All the other parameters in these series of reaction were constant. As shown in Figure 3, as the concentration of the MBA was increased, the water absorbency of the hydrogel composite was decreased $(41,34$ and $17 \mathrm{~g} / \mathrm{g}$ for $0.13,0.32$ and $0.65 \mathrm{mmol}$ of MBA, respectively). Clearly, a higher concentration of crosslinker produces a larger degree of polymer chains branching and generates an additional network. Thereby, with the crosslinker content increasing, the crosslinking density increases. As a result, the network space gets diminished, and less water enters the hydrogel [23]. 


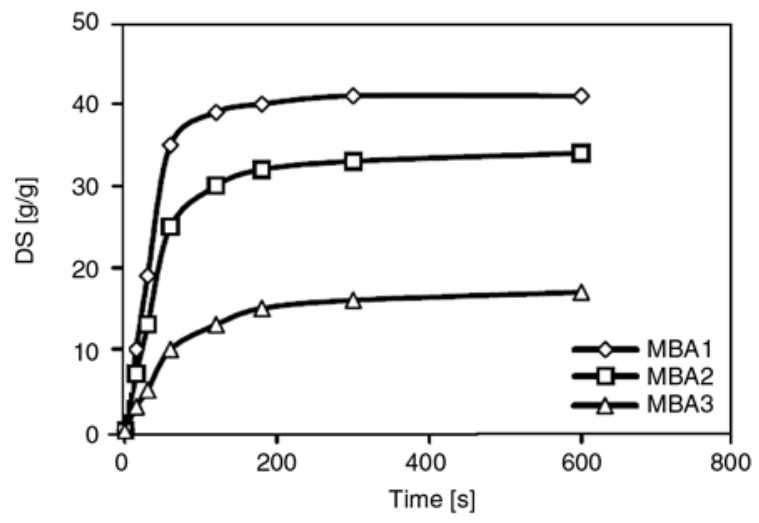

Figure 3. Effect of MBA concentration on the water absorbency and dynamic swelling of hydrogels

\subsection{Release of $\mathrm{KNO}_{3}$ from PAAm hydrogel}

The hydrogels have been evaluated as slow release matrix using potassium nitrate as model agrochemical. Potassium nitrate is used to improve production of crops. It is important to controlled release of agrochemical to diminish amount of active ingredient without reducing efficiency. The release of an active agent from hydrogel matrix may be affected by various parameters. Hydrogel composition and structure as well as \%loading are important parameters. So, in this study we attempt to investigate the effect of MBA concentration and amount of $\mathrm{CaCO}_{3}$ as well as the \%loading on the release kinetic of $\mathrm{KNO}_{3}$ from PAAm matrix. The release kinetic of active agent from loaded hydrogel is closely related to its water sorption kinetic $[6,16]$. The $\%$ loading in various hydrogels was given in Table 1.

\subsubsection{Effect of amount of loading on release of PN}

The release profile of PN from the porous PAAm hydrogel loaded with various amounts of the PN was studied in deionized water. The results are shown in Figure 4. In this section of study, porous PAAm hydrogel, SP3, was immersed in PN solutions with various concentrations of PN $(0.5,1$ and $2 \%$ wt of PN solution). The results show that the loading is increased with increasing the PN concentration in loading medium (\%30 (AA1), \%58 (AA2) and \%85 (AA3) of loading for SP3 hydrogel in solution containing $0.5,1$ and $2 \%$ wt of $\mathrm{PN}$, respectively). Then the dried and loaded hydrogels were immersed in deionized water to study the release profiles. The release profiles indicate that

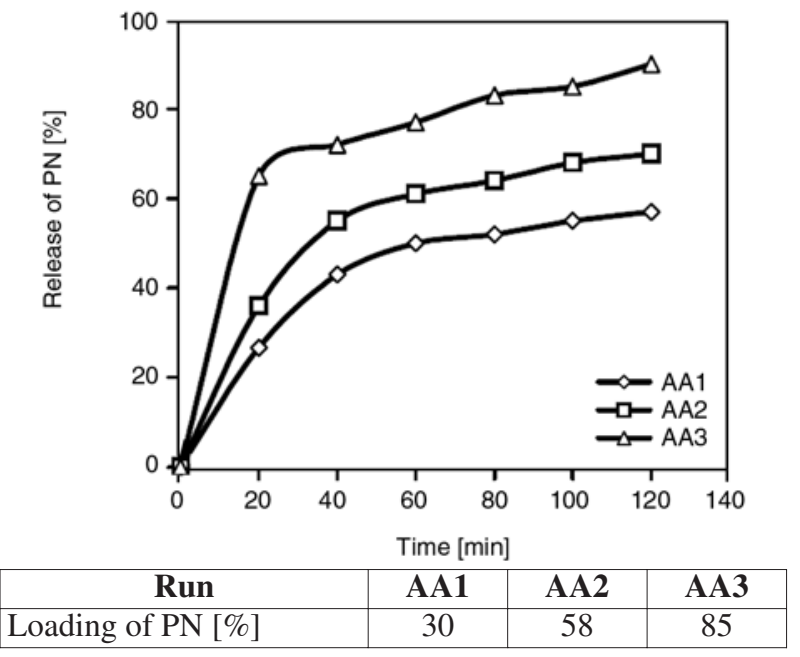

Figure 4. Effect of percentage of loading of PN (SP3 hydrogel) on the release of PN from hydrogel. $\mathrm{AA} 1, \mathrm{AA} 2$ and AA3 are PN loaded SP3 hydrogels containing 30,58 and 85 of \%loading, respectively.

the amount of released PN increases with increasing \%loading of active agent. It is attributed to the larger amount of loading. The larger the initial load, the faster the movement of the solvent front penetrating the surface of the loaded hydrogel $[6,24]$.

\subsubsection{Effect of MBA concentration on the release of $\mathrm{PN}$}

The release of $\mathrm{KNO}_{3}$ from PAAm hydrogels was studied by varying MBA concentration. Figure 5 shows the effect of MBA concentration on the $\mathrm{KNO}_{3}$ release behavior of the hydrogel. The results indicate that the release of active agent depends obviously on the crosslinker concentration. In the

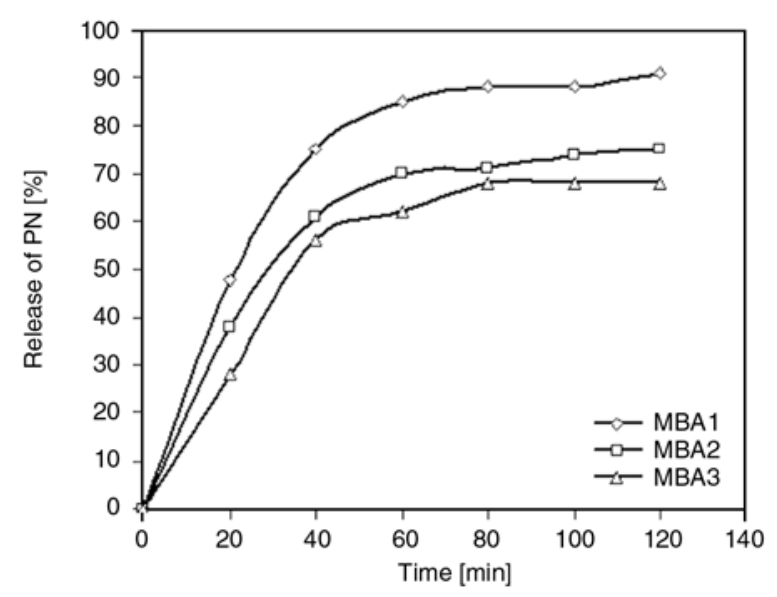

Figure 5. Effect of MBA concentration of the release of PN 
first $40 \mathrm{~min}, 56,61$ and $75 \%$ of $\mathrm{KNO}_{3}$ were released from MBA3, MBA2 and MBA1 hydrogels, respectively. The fast release of $\mathrm{KNO}_{3}$ is due the higher swelling behavior of hydrogel with low concentration of MBA.

\subsubsection{Effect of $\mathrm{CaCO}_{3}$ amount of release of $\mathrm{PN}$}

The $\mathrm{KNO}_{3}$ was entrapped inside the PAAm hydrogels in the swollen state. SP1, SP3 and SP4 were chosen for loading of potassium nitrate. Loading percentages were obtained to be 78,58 and $28 \%$ as determined by conductivity method for SP4, SP3 and SP1, respectively. Figure 6 shows the effect of porosity on the $\mathrm{KNO}_{3}$ release behavior of the hydrogels. The results indicate that the release of active agent depends obviously on the porosity. In the first 40 min, 76 (SP4) and 64 (SP3) percent of $\mathrm{PN}$ were released from porous hydrogels, while in non-porous hydrogel (SP1) the release amount is only 26 percent. The release kinetic of active agent from loaded hydrogel is closely related to its water sorption kinetic $[25,26]$. The fast release of $\mathrm{KNO}_{3}$ in porous hydrogels is due the fast swelling behavior of these polymers.

\section{Conclusions}

Porous PAAm hydrogels were synthesized by solution polymerization of acrylamide in the presence of calcium carbonate particles. Acid treatment of hydrogel- $\mathrm{CaCO}_{3}$ hybrid results in a porous structure in the hydrogels. The porosity of the resulted hydrogels was confirmed using SEM micrographs. Dynamic swelling kinetics of the hydrogels shows that the rate of absorbency for porous hydrogels is

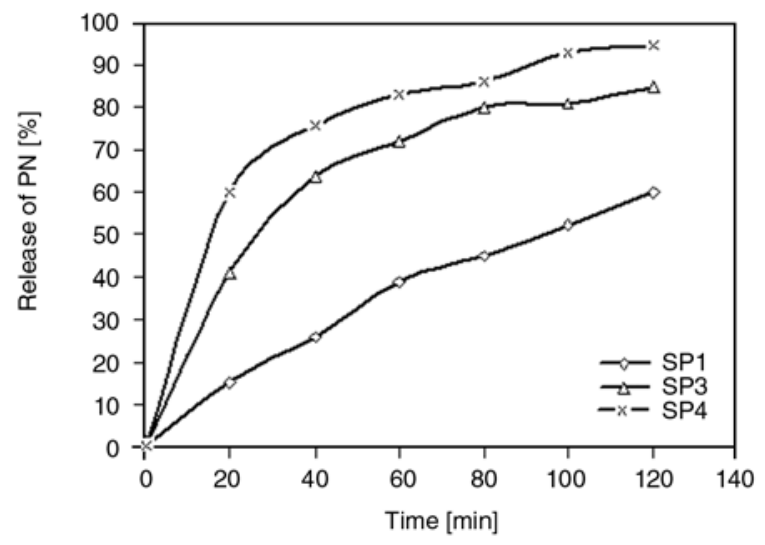

Figure 6. Slow release dependency of hydrogels as a function of used $\mathrm{CaCO}_{3}$ higher than that of non-porous hydrogel. The effect of MBA concentration and $\mathrm{CaCO}_{3}$ amount showed that the water absorbency of the hydrogels is increased with increasing of $\mathrm{CaCO}_{3}$ amount but decreased with increasing MBA concentration. Also, the results of release of PN from hydrogels showed that $\mathrm{CaCO}_{3}$ amount, MBA concentration, and \% loading affect the release of PN from hydrogels.

\section{Acknowledgements}

The authors would like to thank the president of University of Maragheh, Dr Gholam Reza Hojjati, and Research Deputy of University of Maragheh due to their supporting this project. Also, the authors would like to specially thank the referees for their good suggestion to improve the manuscript.

\section{References}

[1] Pó R.: Water-absorbent polymers: A patent survey. Polymer Reviews, 34, 607-662 (1994). DOI: $10.1080 / 15321799408014168$

[2] Dayal U., Mehta S. K., Choudhary M., Jain R. C.: Synthesis of acrylic superabsorbents. Polymer Reviews, 39, 507-525 (1999). DOI: $10.1081 / \mathrm{MC}-100101426$

[3] Liu Q., Rauth A. M., Wu X. Y.: Immobilization and bioactivity of glucose oxidase in hydrogel microspheres formulated by an emulsification-internal gelation-adsorption-polyelectrolyte coating method. International Journal of Pharmaceutics, 339, 148-156 (2007).

DOI: 10.1016/j.ijpharm.2007.02.027

[4] Tonnesen H. H., Karlsen J.: Alginate in drug delivery systems. Drug Development and Industrial Pharmacy, 28, 621-630 (2002). DOI: $\underline{10.1081 / \mathrm{DDC}-120003853}$

[5] Liu M., Liang R., Zhan F., Liu Z., Niu A.: Synthesis of a slow-release and superabsorbent nitrogen fertilizer and its properties. Polymers for Advanced Technologies, 17, 430-438 (2006). DOI: $\underline{10.1002 / \text { pat.720 }}$

[6] Bajpai A. K., Giri A.: Swelling dynamics of a macromolecular hydrophilic network and evaluation of its potential for controlled release of agrochemicals. Reactive and Functional Polymers, 53, 125-141 (2002).

DOI: $10.1016 / \mathrm{S} 1381-5148(02) 00168-2$

[7] Naganagouda N. K., Mulimani V. H.: Gelatin blends with alginate: Gel fibers for $\alpha$-galactosidase immobilization and its application in reduction of nondigestible oligosaccharides in soymilk. Process Biochemistry, 41, 1903-1907 (2006). DOI: $10.1016 /$ j.procbio.2006.03.040 
[8] Sheldon R. A: Enzyme immobilization: The quest for optimum performance. Advanced Synthesis and Catalysis, 349, 1289-1307 (2007).

DOI: $10.1002 /$ adsc. 200700082

[9] Kabiri K., Omidian H., Hashemi S. A., ZohuriaanMehr M. J.: Synthesis of fast-swelling superabsorbent hydrogels: Effect of crosslinker type and concentration on porosity and absorption rate. European Polymer Journal, 39, 1341-1348 (2003).

DOI: $10.1016 / \mathrm{S} 0014-3057(02) 00391-9$

[10] Chen J., Park K.: Synthesis and characterization of superporous hydrogel composites. Journal of Controlled Release, 65, 73-82 (2000).

DOI: $10.1016 / \mathrm{S} 0168-3659(99) 00238-2$

[11] Omidian H., Rocca J. G., Park K.: Advances in superporous hydrogels. Journal of Controlled Release, 102, 3-12 (2005).

DOI: $10.1016 /$ j.jconrel.2004.09.028

[12] Serizawa T., Wakita K., Akashi M.: Rapid deswelling of porous poly $(N$-isopropylacrylamide) hydrogels prepared by incorporation of silica particles. Macromolecules, 35, 10-12 (2002).

DOI: $10.1021 / \mathrm{ma} 011362$

[13] Thomas W. M., Wang D. W.: Acrylamide polymers. in 'Encyclopedia of Polymer Science and Engineering' (eds.: Mark H. F., Bikales N. M.) Vol 1, Wiley, New York, 169-211 (1964).

[14] Saraydin D., Karadag E., Öztop N., Güven O.: Adsorption of bovine serum albumin onto acrylamidemaleic acid hydrogels. Biomaterials, 15, 917-920 (1994).

DOI: 10.1016/0142-9612(94)90117-1

[15] Saraydin D., Karadag E., Cetínkaya S., Güven O.: Preparation of acrylamide/maleic acid hydrogels and their biocompatibility with some biochemical parameters of human serum. Radiation Physics and Chemistry, 46, 1049-1052 (1995).

DOI: $\underline{10.1016 / 0969-806 X(95) 00318-R}$

[16] Abd El-Rehim H. A.: Characterization and possible agricultural application of polyacrylamide/sodium alginate crosslinked hydrogels prepared by ionizing radiation. Journal of Applied Polymer Science, 101, 3572-3580 (2006).

DOI: $10.1002 / a p p .22487$

[17] Abd El-Rehim H. A., Hegazi E. A., Abd El-Mohdy H. L.: Properties of polyacrylamide-based hydrogels prepared by electron beam irradiation for possible use as bioactive controlled delivery matrices. Journal of Applied Polymer Science, 98, 1262-1270 (2005). DOI: 10.1002/app.22167
[18] Lu G. D., Yan Q. Z., Ge C. H.: Preparation of porous polyacrylamide hydrogels by frontal polymerization. Polymer International, 56, 1016-1020 (2007). DOI: $10.1002 /$ pi.2235

[19] Omidian H., Rocca J. G., Park K.: Elastic superporous hydrogel hybrid of polyacrylamide and sodium alginate. Macromolecular Bioscience, 6, 703-710 (2006). DOI: $10.1002 / \mathrm{mabi} .200600062$

[20] Caykara T., Bulut M., Dilsiz N., Akyüz Y.: Macroporous poly(acrylamide) hydrogels: Swelling and shrinking behaviors. Journal of Macromolecular Science Part A: Pure and Applied Chemistry, 43, 889897 (2006).

DOI: $10.1080 / 10601320600653699$

[21] Gemeinhart R. A., Park H., Park K.: Pore structure of superporous hydrogels. Polymers for Advanced Technologies, 11, 617-625 (2000).

DOI: $10.1002 / 1099-1581(200008 / 12) 11: 8 / 12<617::$ AID-PAT12>3.0.CO;2-L

[22] Omidian H., Hashemi S. A., Sammes P. G., Meldrum I.: A model for the swelling of superabsorbent polymers. Polymer, 39, 6697-6704 (1998). DOI: $10.1016 /$ S0032-3861(98)00095-0

[23] Wu J., Lin J., Zhou M., Wei C.: Synthesis and properties of starch-graft-polyacrylamide/clay superabsorbent composite. Macromolecular Rapid Communication, 21, 1032-1034 (2000).

DOI: $10.1002 / 1521-3927(20001001) 21: 15<1032:$ : AID-MARC1032>3.0.CO;2-N

[24] Kim S. W., Bae Y. H., Okano T.: Hydrogels: Swelling, drug loading, and release. Pharmaceutical Research, 9, 283-290 (1992). DOI: $10.1023 / \mathrm{A}: 1015887213431$

[25] Bajpai A. K., Giri A.: Water sorption behaviour of highly swelling (carboxy methylcellulose-g-polyacrylamide) hydrogels and release of potassium nitrate as agrochemical. Carbohydrate Polymers, 53, 271-279 (2003).

DOI: $\underline{10.1016 / \mathrm{S} 0144-8617(03) 00071-7}$

[26] Colombo P., Bettini R., Peppas N. A.: Observation of swelling process and diffusion front position during swelling in hydroxypropyl methyl cellulose (HPMC) matrices containing a soluble drug. Journal of Controlled Release, 61, 83-91 (1999). DOI: $\underline{10.1016 / S 0168-3659(99) 00104-2}$ 